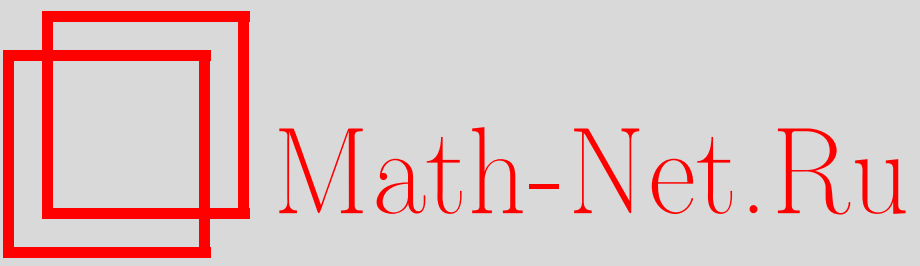

И. А. Козлитин, Математическая модель движения вращающегося снаряда, $\mathrm{Ma-}$ тем. моделирование, 2020, том 32, номер 5, 126-142

DOI: https://doi.org/10.20948/mm-2020-05-07

Использование Общероссийского математического портала Math-Net.Ru подразумевает, что вы прочитали и согласны с пользовательским соглашением

http://www.mathnet.ru/rus/agreement

Параметры загрузки:

IP : 54.205 .225 .156

26 апреля 2023 г., 12:07:14 


\title{
МАТЕМАТИЧЕСКАЯ МОДЕЛЬ ДВИЖЕНИЯ ВРАЩАЮЩЕГОСЯ СНАРЯДА
}

\author{
(C) 2020 2. И.А. Козлитин \\ Институт прикладной математики им. М.В. Келдыша РАН \\ ioannkozlitin@gmail.com
}

DOI: $10.20948 / \mathrm{mm}-2020-05-07$

\begin{abstract}
Предложена полуэмпирическая математическая модель движения центра масс вращающегося снаряда в атмосфере, учитывающая эффекты деривации и изменения лобового сечения сопротивления вследствие изменения угла атаки. Эти эффекты учитываются в модели без детального описания вращения, что позволяет использовать крупный шаг интегрирования и обеспечивает возможность расчета баллистических поправок в реальном масштабе времени. Подробно описан процесс подбора параметров модели для реальных оружейных систем.
\end{abstract}

Ключевые слова: полуэмпирическая модель, внешняя баллистика, деривация, угол атаки, лобовое сечение сопротивления, баллистический вычислитель.

\section{THE MATHEMATICAL MODEL OF MOTION OF A SPINNING PROJECTILE}

\section{I.A. Kozlitin}

Keldysh Institute for Applied Mathematics of Russian Academy of Sciences

The semi-empirical mathematical model for movement of mass center of spinning projectile in atmosphere was proposed. This model takes into account effects of derivation and change of frontal resistance by changing of angle of attack. These effects are taking into account without description of spinning in detail in our model. Therefore big integration step can be used and the opportunity of calculation of ballistic corrections in real time is provided. The procedure for model parameters selection has been described in detail for real weapon systems.

Key words: semi-empirical model, external ballistics, derivation, angle of attack, frontal cross-section resistance, ballistic computer.

Введение. Эффективность применения огнестрельного оружия существенно повышается при наличии развитой системы управления огнем (СУО). Типичной задачей для СУО является обеспечение точной стрельбы с ходу, либо по движущейся цели. Во многих случаях движутся как носитель оружия, так и цель. Для достижения требуемой точности баллистиче- 
ские поправки должны пересчитываться в реальном времени, что накладывает жесткие ограничения на вычислительную сложность алгоритмов, заложенных в баллистическом вычислителе (БВ), входящем в состав СУО.

Рассмотрим различные варианты реализации баллистического вычислителя:

1. Табличный метод. Таблицы стрельбы рассчитываются заранее, а БВ осуществляет их интерполяцию для заданных на его входе параметров цели и атмосферы.

2. Интегрирование системы дифференциальных уравнений внешней баллистики без учета (либо с упрощенным учетом) вращения снаряда. Эффекты, связанные с вращением, учитываются, как в первом способе.

3. Интегрирование полной системы дифференциальных уравнений внешней баллистики с учетом вращения снаряда (модель с 6 степенями свободы или 6DOF-моделирование [1-3]).

Варианты реализации в этом списке перечислены в порядке роста вычислительной сложности алгоритмов БВ. Первый вариант дает максимальную скорость работы БВ, однако в нем сложно учесть взаимное влияние возмущающих факторов друг на друга [2], то есть изменения поправки к одному из параметров при изменении другого параметра (например, при разных температурах атмосферы поправки на изменение атмосферного давления будут тоже разными). Третий вариант предполагает использование очень малого шага интегрирования для детального описания вращения снаряда и поэтому имеет высокую вычислительную сложность [1].

По указанным выше причинам второй вариант реализации БВ представляется наиболее правильным, поскольку допускает использование крупного шага интегрирования и не имеет проблем, присущих первому способу. В этом варианте можно совсем отказаться от использования баллистических таблиц. При этом эффекты, связанные с вращением, учитываются «в среднем», что позволяет сохранить крупный шаг интегрирования, так как нет необходимости детального описания вращения.

Примером реализации такого подхода служит «Modified Point-Mass Trajectory Model» [1], а также 4DOF (4 degrees of freedom) модели [4]. При 4DOF-моделировании наряду с координатами центра масс летящего тела учитывается угол атаки - угол между осью симметрии летящего тела и набегающим потоком воздуха. Процессорное время, требуемое на расчет по таким моделям, существенно меньше по сравнению 6DOF-моделями. Число параметров у 4DOF-моделей также меньше, что облегчает процесс их подбора. Баллистическая модель, рассматриваемая в данной работе, принадлежит к классу 4DOF-моделей. 
Основные уравнения внешней баллистики. Движение центра масс снаряда описывается системой обыкновенных дифференциальных уравнений [5]

$$
\left\{\begin{array}{l}
\frac{d v}{d t}=-g \sin \theta-\frac{F_{T}}{m}, \\
\frac{d \theta}{d t}=-\frac{g}{v} \cos \theta+\frac{F_{N}}{m v} \cos \phi, \\
\frac{d \psi}{d t}=-\frac{F_{N}}{m v \cos \theta} \sin \phi, \\
\frac{d x}{d t}=v \cos \theta \cos \psi, \quad \frac{d y}{d t}=v \sin \theta, \quad \frac{d z}{d t}=v \cos \theta \sin \psi,
\end{array}\right.
$$

где $m$ - масса снаряда, $v$ - модуль его скорости, $\theta$ - угол между вектором скорости и горизонтальной плоскостью XOZ (оси OX и OY перпендикулярны друг другу и лежат в плоскости бросания, ось OZ направлена вправо и перпендикулярна им обоим), $\phi$ - угол прецессии стабилизированного вращением снаряда, $\psi$ - угол между осью ОХ и проекцией вектора скорости на плоскость XOZ, $F_{T}$ - тангенциальная компонента силы сопротивления, $F_{N}$ - её нормальная компонента.

Нормальная $F_{N}$ и тангенциальная $F_{T}$ компоненты аэродинамической силы вычисляются по следующим формулам [5]:

$$
F_{T}=\frac{\pi \rho v^{2} D^{2}}{8} i c_{x}(v / a)\left(1+c_{1} \delta^{2}\right), \quad F_{N}=\frac{\rho v^{2}}{2} D L K_{N}(v / a) \delta\left(1+c_{2} \delta^{2}\right)
$$

где $\rho$ - плотность среды, $D$ - калибр снаряда, $L$ - его длина, $\delta$ - угол нутации (он же - угол атаки) стабилизированного вращением снаряда, $c_{1}, c_{2}-$ эмпирические коэффициенты, зависящие от его формы, $K_{N}(v / a)-$ функция нормальной силы, $a$ - скорость звука, $c_{x}$ - эталонный закон сопротивления, $i$ - коэффициент формы.

В полете снаряд, стабилизированный вращением, под действием силы тяжести совершает прецессионное движение вокруг некоторой динамической оси вращения, которая отклоняется вправо (при правой нарезке) на угол $[5,6]$

$$
\delta_{p}=\frac{\mu m g \cos \theta}{2 \rho v^{3} H K_{M}(v / a)} \frac{d \varphi_{\alpha}}{d t},
$$


где $\rho$ - плотность среды, $H$ - плечо аэродинамической силы, $d \varphi_{\alpha} / d t-$ угловая скорость вращения снаряда относительно вектора скорости, $\mu-$ коэффициент, связанный с распределением массы внутри снаряда, $K_{M}(v / a)-$ функция опрокидывающего момента. Отклонение динамической оси вращения на угол $\delta_{p} \neq 0$ от вектора скорости приводит к возникновению нескомпенсированной нормальной силы, действие которой является причиной эффекта деривации.

Вращение снаряда в полете постепенно замедляется вследствие действия силы трения. Этот процесс описывается уравнением $[5,6]$

$$
\frac{d}{d t} \frac{d \varphi_{a}}{d t}=-4 \rho v \frac{D L}{\mu m} K_{r}(v / a) \omega_{\varphi}
$$

где $d \varphi_{\alpha} / d t$ - угловая скорость вращения снаряда вокруг вектора скорости, $\omega_{\varphi}$ - угловая скорость вращения вокруг собственной оси, а $K_{r}(v / a)-$ функция поверхностного трения.

Начальную угловую скорость вращения снаряда вокруг собственной оси $\omega_{\varphi 0}$ можно вычислить, зная его калибр $D$, длину хода нарезов в калибpax $\eta$ и начальную скорость снаряда $v_{0}[5]$

$$
\omega_{\varphi 0}=\frac{2 \pi}{\eta D} v_{0} .
$$

В [5] для описания движения центра масс снаряда с учетом деривации используются следующие упрощающие предположения:

1. Нормальная компонента силы сопротивления лежит в плоскости сопротивления (плоскость, натянутая на вектор скорости и ось симметрии снаряда), поворачиваясь вместе с этой плоскостью со скоростью прецессии. При рассмотрении вращения «в среднем» вводится нескомпенсированная нормальная сила $F_{N}$, направленная перпендикулярно мгновенной плоскости бросания (плоскость, натянутая на вектора скорости и ускорения свободного падения). Для введения нескомпенсированной нормальной силы в модель в формулах (1) берут угол прецессии $\phi=\pi / 2$.

2. Угол $\psi$ отклонения траектории снаряда от первоначальной линии бросания, вызванный деривацией, является малым углом, поэтому $\operatorname{tg} \psi \simeq \sin \psi \simeq \psi, \cos \psi \simeq 1$ и т.п.

3. Скорость вращения снаряда вокруг собственной оси существенно больше скорости прецессии. Поэтому можно пренебречь разницей между 
угловой скоростью вращения снаряда вокруг вектора скорости $d \varphi_{\alpha} / d t$, включающей также прецессионное вращение, и вокруг собственной оси $\omega_{\varphi}$, т.е. $d \varphi_{\alpha} / d t=\omega_{\varphi}$.

4. Угол нутации $\delta$ в выражении для нормальной силы $F_{N}$ берется равным углу отклонения динамической оси вращения от вектора скорости $-\delta_{p}$ (знак минус вызван тем, что нормальная компонента аэродинамической силы $F_{N}$ направлена противоположно оси $\mathrm{OZ}$ ), что соответствует учету эффектов вращения «в среднем», когда в качестве среднего угла нутации используется $-\delta_{p}$.

5. Для снарядов, устойчивых в полете, угол нутации $\delta$ мал, поэтому можно считать, что нормальная компонента аэродинамической силы $F_{N}$ линейно зависит от угла нутации $\delta$, то есть $c_{2}=0$.

6. При малых углах нутации опрокидывающий момент можно считать линейным относительно угла нутации $\delta$.

7. Влияние демпфирующего момента для стабилизированных вращением снарядов мало по сравнению с опрокидывающим моментом, поэтому демпфирующим моментом можно пренебречь.

Два последних предположения применяются при выводе формулы (3).

В нашей модели угол отклонения динамической оси вращения $-\delta_{p}$ используется в качестве среднего угла нутации (угла атаки) $\delta$ не только в формуле для $F_{N}$, но и в выражении для $F_{T}$, что не было сделано в $[1,5]$. Таким образом, учитывается «в среднем» изменение лобового сечения сопротивления вследствие изменения угла атаки.

С учетом указанных выше упрощающих предположений система уравнений (1) после подстановки формул (2) и перехода к новой независимой переменной $x$ примет следующий вид:

$$
\left\{\begin{array}{l}
\frac{d u}{d x}=-\frac{\pi \rho v D^{2}}{8 m} i c_{x}(v / a)\left(1+c_{1} \delta^{2}\right), \\
\frac{d \gamma}{d x}=-\frac{g}{u^{2}}, \frac{d y}{d x}=\gamma, \quad \frac{d z}{d x}=\xi, \quad \frac{d t}{d x}=\frac{1}{u}, \\
\frac{d \xi}{d x}=B_{N M}(v / a) \frac{v_{\varphi}}{v^{2} u}, \quad \frac{d v_{\varphi}}{d x}=-\rho \sqrt{1+\gamma^{2}} B_{r}(v / a) v_{\varphi}, \\
v=u \sqrt{1+\gamma^{2}}, \quad \delta=\frac{v_{\varphi}}{\rho v^{3} \sqrt{1+\gamma^{2}}} B_{\delta}(v / a) .
\end{array}\right.
$$


Здесь

$$
\begin{aligned}
& u=v \cos \theta, \quad \xi=\operatorname{tg} \psi, \quad \gamma=\operatorname{tg} \theta, \quad v_{\varphi}=\frac{\eta D}{2 \pi} \omega_{\varphi}, \\
& B_{N M}(v / a)=\frac{\pi g}{2} \frac{\mu L}{\eta H} K_{N M}(v / a), \quad K_{N M}(v / a)=K_{N}(v / a) K_{M}^{-1}(v / a), \\
& B_{\delta}(v / a)=\frac{\pi g \mu m}{D H \eta} K_{M}^{-1}(v / a), \quad B_{r}(v / a)=\frac{4 D L}{\mu m} K_{r}(v / a) .
\end{aligned}
$$

Систему дифференциальных уравнений (6) необходимо дополнить следующими начальными условиями:

$$
\begin{aligned}
& u(0)=v_{0} \cos \theta_{0}, \quad \gamma(0)=\operatorname{tg} \theta_{0}, y(0)=0, \\
& z(0)=0, \quad t(0)=0, \quad \xi(0)=0, \quad v_{\varphi}(0)=v_{0},
\end{aligned}
$$

где $v_{0}$ - начальная скорость, $\theta_{0}$ - угол бросания.

Для вычисления угла бросания $\theta_{0}$ для некоторой дальности $l$ необходимо поставить граничное условие $y(l)=0$. В результате получается краевая задача для системы дифференциальных уравнений (6). Ее решение даст искомый угол $\theta_{0}$.

Построение полуэмпирической модели. Для расчета траектории полета тела по формулам (6)-(8) необходимо знание всех входящих в них параметров и функциональных зависимостей. При этом функции $c_{x}(v / a)$, $K_{M}(v / a), K_{N M}(v / a), K_{r}(v / a)$ уникальны для данной формы летящего тела и не вполне применимы к телам иной формы. Отчасти эта проблема решается путем построения так называемых эталонных зависимостей, пригодных для некоторого класса тел похожей формы, и добавления в модель подгоночных параметров, необходимых для согласования модели с экспериментом. В случае с законом сопротивления $c_{x}(v / a)$ таким параметром является коэффициент формы $i$.

В литературе имеются эталонные зависимости лишь для функций $c_{x}(v / a)$ и $K_{N M}(v / a)$ [3,7-10]. Остальные функции приходится заменять аппроксимациями, зависящими от свободных параметров, которые подбираются путем согласования с экспериментальными данными (либо результатами газодинамических расчетов). В результате модель становится полуэмпирической. Тестовые расчеты показали, что использование $K_{N M}(v / a)$ из [3] не дает повышения точности расчетов, поэтому в данной модели эта эталонная зависимость не применяется. 
На практике вместо функций $K_{M}(v / a), K_{N M}(v / a), K_{r}(v / a)$ удобнее рассматривать функции $B_{N M}(v / a), B_{r}(v / a), B_{\delta}(v / a)$. Аппроксимируем их следующим образом:

$$
B_{N M}(v / a)=\beta_{n m}, \quad B_{r}(v / a)=\beta_{r}(v / a)^{\beta_{\alpha}-1}, \quad B_{\delta}(v / a)=\beta_{\delta}(v / a)^{\beta_{\gamma}},
$$

где $\beta_{n m}, \beta_{r}, \beta_{\alpha}, \beta_{\delta}, \beta_{\gamma}$ - параметры модели.

Такая форма аппроксимаций выбрана на основании следующих соображений:

1. Функция $B_{N M}(v / a) \sim K_{N M}(v / a)$ согласно (7). В свою очередь, $K_{N M}(v / a)$ нередко аппроксимируется константой [5], поэтому в (9) $B_{N M}(v / a)$ также аппроксимирована константой.

2. Замедление вращения снаряда описывается уравнением (4). При вычислении деривации в [5] правая часть (4) предполагается линейно зависящей от угловой скорости вращения вокруг собственной оси $\omega_{\varphi}$, что позволяет проинтегрировать уравнение (4) аналитически. Согласно (7) и (9) из этого следует, что $B_{r}(v / a) \sim(v / a)^{-1}$. В нашей модели в показатель степени добавлен поправочный коэффициент $\beta_{\alpha}$, что позволяет устранить произвол в выборе показателя степени и тем самым увеличить физическую точность модели.

3. Выражение для $B_{\delta}$ аналогично выражению для $B_{r}$ как некоторому обобщению константной аппроксимации.

Данные аппроксимации в совокупности и изложенный ниже метод определения их параметров ранее никем не рассматривались и поэтому составляют новизну работы.

Подставим величину $\delta$ из (6) в первое уравнение (6) с учетом (9). В результате получим

$$
\frac{d u}{d x}=-\frac{\pi \rho v D^{2}}{8 m} i c_{x}(v / a)\left(1+c_{1} \beta_{\delta}^{2}\left(\frac{v_{\varphi}}{\rho v^{3} \sqrt{1+\gamma^{2}}}(v / a)^{\beta_{\gamma}}\right)^{2}\right) .
$$

Поскольку параметры модели $c_{1}$ и $\beta_{\delta}$ входят только в (10) и не входят в другие уравнения из (6), то при выполнении соотношения $c_{1} \beta_{\delta}^{2}=$ const поведение модели не зависит от конкретных значений $c_{1}$ и $\beta_{\delta}$, так что без ограничения общности можно считать $c_{1}=1$, тем самым исключив параметр $c_{1}$ из рассмотрения.

Коэффициент формы $i$ совместно с параметрами (9) образуют полный набор параметров модели, которые можно объединить в вектор

$$
\mathbf{p}=\left(i, \beta_{\delta}, \beta_{\gamma}, \beta_{n m}, \beta_{r}, \beta_{\alpha}\right) .
$$


Оптимизация параметров модели. Назовем вектор параметров модели $\mathbf{p}_{\text {opt }}$ оптимальным, если

$$
\mathbf{p}_{\text {opt }}=\arg \min R(\mathbf{p}) \text {, }
$$

где

$$
R(\mathbf{p})=\frac{1}{N} \sum_{j}\left(\frac{y^{2}\left(\mathbf{p}, l_{j}, \theta_{0 j}\right)}{1+\gamma^{2}\left(\mathbf{p}, l_{j}, \theta_{0 j}\right)}+\left(z\left(\mathbf{p}, l_{j}, \theta_{0 j}\right)-z_{d}\left(l_{j}\right)\right)^{2}\right)
$$

характеризует отклонение баллистических поправок, вычисленных по модели, от табличных. Здесь $N$ - число значений дальности в таблице стрельбы, $l_{j}$ - табличная дальность, $z_{d}\left(l_{j}\right)$ - табличное значение деривации при заданной дальности, $y\left(\mathbf{p}, l_{j}, \theta_{0 j}\right), z\left(\mathbf{p}, l_{j}, \theta_{0 j}\right), \gamma\left(\mathbf{p}, l_{j}, \theta_{0 j}\right)$ - расчетные (вычисленные по модели) высота полета, боковое отклонение и тангенс угла падения тела (угол рассчитывается в плоскости ХОY и боковое отклонение не учитывается в силу его малости) соответственно при $x=l_{j}$, наборе параметров модели $\mathbf{p}$ и угле бросания $\theta_{0 j}$, взятом из таблиц стрельбы. Учет угла падения в (13) позволяет корректно оценить ошибку модели как при настильной, так и при навесной траектории полета тела.

В формуле (13) можно выделить два слагаемых, первое из которых отвечает за погрешность при определении углов бросания, а второе - за погрешность при вычислении деривации. С другой стороны, 6 параметров модели, входящих в (11), можно разделить на две группы: первые 3 параметра $i, \beta_{\delta}, \beta_{\gamma}$ (назовем их траекторными) отвечают непосредственно за траекторию полета в плоскости бросания, а оставшиеся параметры $\beta_{n m}, \beta_{r}, \beta_{\alpha}$ (назовем их деривационными) - за боковое отклонение (деривацию). При $\beta_{\delta}=0$ деривационные параметры не оказывают на траекторию полета в плоскости бросания никакого влияния. Если $\beta_{\delta} \neq 0$, то имеется косвенная зависимость через угол нутации $\delta$, однако она небольшая по сравнению с влиянием траекторных параметров.

Данное обстоятельство позволяет подбирать траекторные и деривационные параметры отдельно друг от друга, используя первое и второе слагаемые в (13) соответственно. С другой стороны, поскольку косвенная зависимость этих групп параметров существует, то это необходимо учитывать при их подборе. Например, определение деривационных параметров невозможно без предварительного подбора траекторных параметров, а определение траекторных параметров $\beta_{\delta}, \beta_{\gamma}$ невозможно без знания деривационных параметров. 
Опираясь на сказанное выше, можно предложить следующую пошаговую процедуру решения задачи оптимизации (12):

1. Определить траекторные параметры в первом приближении, пренебрегая эффектом изменения лобового сопротивления вследствие изменения угла атаки (т.е. положить $\beta_{\delta}=0, \beta_{\gamma}=0$ ). Исключение из рассмотрения параметров $\beta_{\delta}$ и $\beta_{\gamma}$ компенсируется введением зависимости коэффициента формы от угла бросания $i\left(\theta_{0}\right)$.

2. Определить деривационные параметры в первом приближении, не меняя траекторных параметров («заморозить» траекторные параметры).

3. Определить траекторные параметры во втором приближении, не меняя деривационных («заморозить» деривационные параметры). Зависимость коэффициента формы от угла бросания $i\left(\theta_{0}\right)$ при этом исключается из модели, а $\beta_{\delta}$ и $\beta_{\gamma}$ включаются в нее.

4. Уточнить все параметры модели, решив исходную задачу оптимизации (12), не прибегая при этом к «заморозке» параметров. В качестве начального приближения берется результат предыдущего шага.

Рассмотрим этот процесс более подробно. Шаги 1, 2 и 4 состоят из одного этапа, 3 шаг состоит из двух этапов. Каждый шаг соответствует некоторой стадии алгоритма, каждый этап - решению вспомогательной задачи минимизации.

Ha первом этапе строится зависимость коэффициента формы $i$ от угла бросания $\theta_{0}$. Для этого для каждой дальности $l$ и табличного угла бросания $\theta_{0}(l)$ решается нелинейное уравнение относительно $i$

$$
y\left(\mathbf{p}(i), l, \theta_{0}(l)\right)=0,
$$

где $\mathbf{p}(i)=(i, 0,0,0,0,0)$, то есть все параметры модели, кроме коэффициента формы, берутся нулевыми. В результате получается зависимость $i\left(\theta_{0}\right)$.

На втором этапе определяются параметры $\beta_{n m}, \beta_{r}, \beta_{\alpha}$, отвечающие за расчет деривации. Для этого по этим параметрам минимизируется величина

$$
R_{1}\left(\beta_{n m}, \beta_{r}, \beta_{\alpha}\right)=\sum_{j}\left(z\left(\left(i\left(\theta_{0 j}\right), 0,0, \beta_{n m}, \beta_{r}, \beta_{\alpha}\right), l_{j}, \theta_{0 j}\right)-z_{d}\left(l_{j}\right)\right)^{2} .
$$

Заметим, что в точке минимума $\partial R_{1} / \partial \beta_{n m}=0$. Из этой формулы следует, что

$$
\beta_{n m}=\sum_{j} z_{j} z_{d}\left(l_{j}\right)\left(\sum_{j} z_{j}^{2}\right)^{-1},
$$

где $z_{j}=z\left(\left(i\left(\theta_{0 j}\right), 0,0,1, \beta_{r}, \beta_{\alpha}\right), l_{j}, \theta_{0 j}\right)$, поэтому 


$$
R_{1}\left(\beta_{n m}, \beta_{r}, \beta_{\alpha}\right)=R_{1}\left(\sum_{j} z_{j} z_{d}\left(l_{j}\right)\left(\sum_{j} z_{j}^{2}\right)^{-1}, \beta_{r}, \beta_{\alpha}\right)=R_{2}\left(\beta_{r}, \beta_{\alpha}\right) .
$$

Для минимизации $R_{2}\left(\beta_{r}, \beta_{\alpha}\right)$ сначала минимизируем $R_{2}\left(\beta_{r}, \beta_{\alpha}\right)$ при $\beta_{\alpha} \equiv 0$, то есть определим

$$
\beta_{r}^{(0)}=\underset{\beta_{r}}{\arg \min } R_{2}\left(\beta_{r}, 0\right) .
$$

В качестве начального приближения для $\beta_{r}^{(0)}$ берется 0.

Далее, получив $\beta_{r}^{(0)}$, решаем более сложную задачу

$$
\left(\beta_{r}^{(1)}, \beta_{\alpha}^{(1)}\right)=\underset{\left(\beta_{r}, \beta_{\alpha}\right)}{\arg \min } R_{2}\left(\beta_{r}, \beta_{\alpha}\right),
$$

выбрав в качестве начального приближения вектор $\left(\beta_{r}^{(0)}, 0\right)$.

Для определения $\beta_{n m}^{(1)}$ при известных $\beta_{r}^{(1)}$ и $\beta_{\alpha}^{(1)}$ используем (16). На этом завершается второй этап решения исходной задачи.

На третьем этапе определяем траекторные параметры $i, \beta_{\delta}, \beta_{\gamma}$ при «замороженных» $\beta_{n m}, \beta_{r}, \beta_{\alpha}$, найденных на втором этапе. Для этого рассмотрим

$$
\mathbf{p}\left(\beta_{\delta}, \beta_{\gamma}\right)=\left(i\left(\beta_{\delta}, \beta_{\gamma}\right), \beta_{\delta}, \beta_{\gamma}, \beta_{n m}^{(1)}, \beta_{r}^{(1)}, \beta_{\alpha}^{(1)}\right),
$$

где

$$
i\left(\beta_{\delta}, \beta_{\gamma}\right)=\underset{i}{\arg \min } R_{X Y}\left(i, \beta_{\delta}, \beta_{\gamma}, \beta_{n m}^{(1)}, \beta_{r}^{(1)}, \beta_{\alpha}^{(1)}\right) .
$$

Здесь

$$
R_{X Y}(\mathbf{p})=\frac{1}{N} \sum_{j} \frac{y^{2}\left(\mathbf{p}, l_{j}, \theta_{0 j}\right)}{1+\gamma^{2}\left(\mathbf{p}, l_{j}, \theta_{0 j}\right)} .
$$

Далее определим

$$
\mathbf{p}_{0}=\underset{\mathbf{p}}{\arg \min } R_{X Y}(\mathbf{p}),
$$

где $\mathbf{p}$ принадлежат множеству (20), а в качестве начального приближения берем $\beta_{\delta}=1$ и $\beta_{\gamma}=0$.

В результате решения задачи (23) получим вектор

$$
\mathbf{p}_{0}=\left(i^{(1)}, \beta_{\delta}^{(1)}, \beta_{\gamma}^{(1)}, \beta_{n m}^{(1)}, \beta_{r}^{(1)}, \beta_{\alpha}^{(1)}\right),
$$

где $\beta_{\delta}^{(1)}, \beta_{\gamma}^{(1)}$ - параметры, обеспечивающие минимум (23). 
Для уменьшения вычислительной сложности задачи (23) суммирование в (22) следует вести не для всех дальностей из таблицы, а лишь для некоторых опорных дальностей. В данной работе использовались два значения: первое - максимальная дальность из таблицы, второе - половина максимальной дальности.

На четвертом этапе вновь решается задача (23) уже по всем дальностям, а не только по опорным, с «замороженными» параметрами $\beta_{n m}^{(1)}, \beta_{r}^{(1)}$, $\beta_{\alpha}^{(1)}$ относительно трёх переменных: $i^{(1)}, \beta_{\delta}^{(1)}, \beta_{\gamma}^{(1)}$. При этом в качестве начального приближения используется $\mathbf{p}_{0}$. В результате минимизации на этом этапе получаем вектор $\mathbf{p}_{1}$.

На пятом этапе решается исходная задача (12) с начальным приближением $\mathbf{p}_{1}$.

Вычисление углов бросания по углам прицеливания. Для подбора параметров баллистической модели необходимо для каждой дальности $l$ знать соответствующий угол бросания $\theta_{0}(l)$. К сожалению, нередко в таблицах стрельбы не приводится углов бросания. Вместо них указываются углы прицеливания $\alpha(l)$, которые зависят не только от углов бросания $\theta_{0}(l)$, но также от угла вылета пули (снаряда) и особенностей конструкции прицела. Поэтому возникает задача определения углов бросания по углам прицеливания.

Из простых геометрических соображений следует, что

$$
y(\mathbf{p}(i), l, \alpha(l)+\Delta \alpha)=h,
$$

где $i$ - коэффициент формы, $\Delta \alpha$ - угол между линиями прицеливания и бросания, а $h$ - эффективное смещение по вертикали оси ствола относительно оптической оси прицела. Остальные обозначения соответствуют формуле (14).

Определим $i, \Delta \alpha, h$ из решения задачи оптимизации

$$
(i, \Delta \alpha, h)=\underset{(i, \Delta \theta, h)}{\arg \min } \sum_{j}\left(y\left(\mathbf{p}(i), l_{j}, \alpha\left(l_{j}\right)+\Delta \alpha\right)-h\right)^{2} .
$$

Из множества дальностей, по которым минимизируется (26), следует исключить самые малые дальности, так как для них угол прицеливания $\alpha(l)$ известен со значительной относительной погрешностью, а также большие дальности, при которых становится существенной зависимость коэффициента формы $i$ от угла бросания $\theta_{0}$. В наших расчетах использовались дальности из диапазона $150 \ldots 600$ м. 
После определения $\Delta \alpha, h$ вычисление угла бросания $\theta_{0}(l)$ для каждой дальности $l$ осуществляется следующим образом:

1. Уравнение (25) решается относительно коэффициента формы $i$.

2. Решается уравнение $y\left(\mathbf{p}(i), l, \theta_{0}\right)=0$ относительно $\theta_{0}$ с коэффициентом формы $i$, полученным на первом шаге.

Этот алгоритм правильно работает при дальностях $l>100$ м. При меньших дальностях из-за большой относительной погрешности угла прицеливания на первом шаге получается недостоверная величина коэффициента формы $i$. Поэтому при выполнении второго шага алгоритма для этих дальностей разумно использовать коэффициент формы $i$, полученный при больших дальностях.

Определение оптимального числа параметров модели. Во многих случаях (например, при расчете баллистики стрелкового оружия) число параметров модели избыточно. Недостаточная точность исходных данных не позволяет надежно определить все параметры. Во избежание эффекта переобучения следует наращивать число параметров модели постепенно.

Сначала рассматривается наиболее простой вариант, учитывающий лишь самые основные физические факторы. Затем в модель включаются дополнительный фактор (например, учет угла атаки или замедления вращения в полете). Если это приводит к статистически значимому уменьшению погрешности согласования с экспериментальными данными, то этот фактор добавляется в модель, иначе он далее не учитывается.

Для оценки статистической значимости фактора использовался $F$ критерий Фишера [11], основанный на анализе остаточных дисперсий. Рассмотрим его применение для оценки статистической значимости учета угла атаки при подборе параметров баллистической модели.

Рассмотрим две остаточные дисперсии

$$
D_{1}(i)=\frac{N}{N-1} R_{X Y}\left(\mathbf{p}_{D 1}(i)\right), \quad D_{2}\left(i, \beta_{\delta}, \beta_{\gamma}\right)=\frac{N}{N-3} R_{X Y}\left(\mathbf{p}_{D 2}\left(i, \beta_{\delta}, \beta_{\gamma}\right)\right),
$$

где $\mathbf{p}_{D 1}(i)=\left(i, 0,0, \beta_{n m}, \beta_{r}, \beta_{\alpha}\right)$ и $\mathbf{p}_{D 2}\left(i, \beta_{\delta}, \beta_{\gamma}\right)=\left(i, \beta_{\delta}, \beta_{\gamma}, \beta_{n m}, \beta_{r}, \beta_{\alpha}\right)$. Первая из них $\left(D_{1}\right)$ соответствует случаю пренебрежения зависимостью лобового сопротивления от угла атаки, вторая $\left(D_{2}\right)$ - её учету. Минимизируем величины $D_{1}$ и $D_{2}$ по их параметрам.

Если

$$
D_{1} / D_{2} \geq F_{\alpha}(N-1, N-3),
$$

где $F_{\alpha}$ - квантиль распределения Фишера для уровня значимости $\alpha$, то согласно критерию Фишера уменьшение остаточной дисперсии $D_{2}$ по срав- 
нению с $D_{1}$ является статистически значимым на уровне значимости $\alpha$ и принимается гипотеза о необходимости учета угла атаки, иначе - отвергается. Аналогично можно оценить статистическую значимость других факторов: замедления вращения снаряда в полете, необходимости введения в модель коэффициентов $\beta_{\alpha}$ и $\beta_{\gamma}$ и др.

Сокращение числа используемых параметров модели способствует увеличению устойчивости процесса подбора параметров, сокращает время подбора и позволяет избежать ситуации, когда результат подбора параметров модели противоречит ее физической сути.

Тестовые расчеты. Рассмотрим процесс подбора параметров модели на примере 30-мм автоматического гранатомета АГС-17 [12].

На первом этапе необходимо для каждой дальности $l$ по углу прицеливания $\alpha(l)$ вычислить угол бросания $\theta_{0}(l)$. Решение задачи (26) дает параметры $\Delta \alpha, h$, а также первичную оценку для коэффициента формы $i$ :

$$
i=1.8172, \Delta \alpha=3.3536, h=-0.1788
$$

при этом дальности $l$ при решении задачи минимизации взяты из диапазона $150 \ldots 600$ м. Используя вычисленные параметры прицела можно рассчитать углы бросания $\theta_{0}(l)$ с помощью алгоритма, описанного выше. Результаты приведены в табл. 1.

Таблица 1. Основная таблица стрельбы для АГС-17.

\begin{tabular}{|c|c|c|c|c|c|c|c|c|}
\hline$l$ & $\alpha(l)$ & $\theta_{0}(l)$ & $l$ & $\alpha(l)$ & $\theta_{0}(l)$ & $l$ & $\alpha(l)$ & $\theta_{0}(l)$ \\
\hline $\mathbf{5 0}$ & 2,220 & 6,94 & $\mathbf{9 0 0}$ & 164,17 & 167,72 & $\mathbf{1 7 3 0}$ & 666,67 & 669,82 \\
\hline $\mathbf{1 0 0}$ & 9,440 & 14,08 & $\mathbf{9 5 0}$ & 177,5 & 181,04 & $\mathbf{1 7 0 0}$ & 768,33 & 771,52 \\
\hline $\mathbf{1 5 0}$ & 16,94 & 21,44 & $\mathbf{1 0 0 0}$ & 191,39 & 194,92 & $\mathbf{1 6 5 0}$ & 833,33 & 836,60 \\
\hline $\mathbf{2 0 0}$ & 24,72 & 28,93 & $\mathbf{1 0 5 0}$ & 205,83 & 209,36 & $\mathbf{1 6 0 0}$ & 879,17 & 882,45 \\
\hline $\mathbf{2 5 0}$ & 32,78 & 36,82 & $\mathbf{1 1 0 0}$ & 221,11 & 224,63 & $\mathbf{1 5 5 0}$ & 916,67 & 919,97 \\
\hline $\mathbf{3 0 0}$ & 41,11 & 45,04 & $\mathbf{1 1 5 0}$ & 237,22 & 240,74 & $\mathbf{1 5 0 0}$ & 949,17 & 952,48 \\
\hline $\mathbf{3 5 0}$ & 49,44 & 53,29 & $\mathbf{1 2 0 0}$ & 254,44 & 257,95 & $\mathbf{1 4 5 0}$ & 978,33 & 981,65 \\
\hline $\mathbf{4 0 0}$ & 58,06 & 61,84 & $\mathbf{1 2 5 0}$ & 272,78 & 276,28 & $\mathbf{1 4 0 0}$ & 1005,00 & 1008,32 \\
\hline $\mathbf{4 5 0}$ & 66,94 & 70,68 & $\mathbf{1 3 0 0}$ & 292,22 & 295,73 & $\mathbf{1 3 5 0}$ & 1029,44 & 1032,77 \\
\hline $\mathbf{5 0 0}$ & 76,11 & 79,81 & $\mathbf{1 3 5 0}$ & 312,78 & 316,28 & $\mathbf{1 3 0 0}$ & 1052,22 & 1055,55 \\
\hline $\mathbf{5 5 0}$ & 85,56 & 89,22 & $\mathbf{1 4 0 0}$ & 334,72 & 338,22 & $\mathbf{1 2 5 0}$ & 1073,61 & 1076,94 \\
\hline $\mathbf{6 0 0}$ & 95,56 & 99,20 & $\mathbf{1 4 5 0}$ & 358,61 & 362,11 & $\mathbf{1 2 0 0}$ & 1093,89 & 1097,22 \\
\hline $\mathbf{6 5 0}$ & 105,83 & 109,45 & $\mathbf{1 5 0 0}$ & 385,56 & 389,06 & $\mathbf{1 1 5 0}$ & 1113,06 & 1116,39 \\
\hline $\mathbf{7 0 0}$ & 116,39 & 119,99 & $\mathbf{1 5 5 0}$ & 416,67 & 420,17 & $\mathbf{1 1 0 0}$ & 1131,39 & 1134,73 \\
\hline $\mathbf{7 5 0}$ & 127,5 & 131,09 & $\mathbf{1 6 0 0}$ & 453,06 & 456,57 & $\mathbf{1 0 5 0}$ & 1149,17 & 1152,50 \\
\hline $\mathbf{8 0 0}$ & 139,17 & 142,74 & $\mathbf{1 6 5 0}$ & 496,39 & 499,93 & $\mathbf{1 0 0 0}$ & 1166,67 & 1170,01 \\
\hline $\mathbf{8 5 0}$ & 151,39 & 154,95 & $\mathbf{1 7 0 0}$ & 556,67 & 560,22 & & & \\
\hline
\end{tabular}


В процессе вычисления углов прицеливания по углам бросания попутно определяется зависимость $i\left(\theta_{0}\right)$, то есть выполняется первый этап алгоритма подбора параметров баллистической модели. Графически эта зависимость приведена на рис.1 (пунктирная линия).

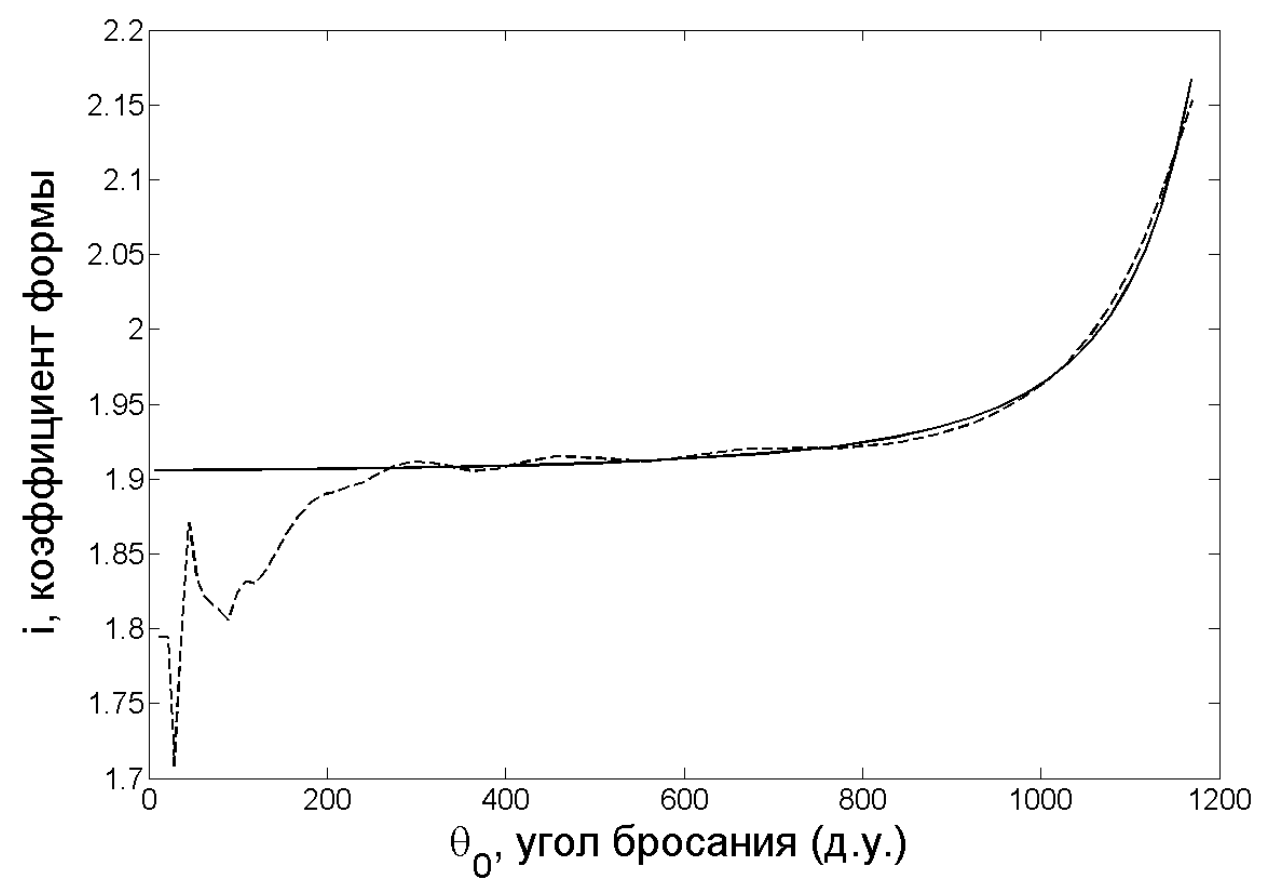

Рис.1. Зависимость коэффициента формы от угла бросания. Сплошная линия модельная кривая, пунктирная - построена по данным из таблиц стрельбы.

Из рис.1 видно, что при углах бросания менее 200 д.у. коэффициент формы испытывает нефизичные осцилляции, что объясняется большой относительной погрешностью табличного угла прицеливания. Далее колебания коэффициента формы уменьшаются, а начиная с угла бросания в 700 д.у. виден его быстрый рост при увеличении угла бросания. Этот рост является следствием того, что с ростом угла бросания средний угол атаки вращающейся гранаты также увеличивается и ее сечение сопротивления возрастает.

На втором этапе оцениваются параметры модели, связанные с боковым отклонением траектории гранаты (деривацией). На этом этапе расчет идет в две стадии. На первой стадии подбирается $\beta_{r}$ при условии $\beta_{\alpha} \equiv 0$. В результате расчета было получено

$$
\beta_{r}^{(0)}=0.0185741 .
$$

На второй стадии используется начальное приближение с первой стадии, а условие $\beta_{\alpha} \equiv 0$ снимается. Решение задачи минимизации дает

$$
\beta_{r}^{(1)}=0.00594269, \quad \beta_{n m}^{(1)}=0.0643147, \quad \beta_{\alpha}^{(1)}=-0.57971 .
$$


На третьем этапе получаются оценки для основных параметров модели

$$
i^{(1)}=1.90841, \quad \beta_{\delta}^{(1)}=111.086, \quad \beta_{\gamma}^{(1)}=-0.723247 .
$$

На четвертом этапе эти оценки параметров уточняются

$$
i^{(2)}=1.90608, \quad \beta_{\delta}^{(2)}=889.709, \quad \beta_{\gamma}^{(2)}=0.225377 .
$$

Наконец, после пятого этапа получим окончательно

$$
\begin{aligned}
& \beta_{r}=0.00454974, \quad \beta_{n m}=0.0620063, \quad \beta_{\alpha}=-0.526863, \\
& i=1.90507, \beta_{\delta}=926.604, \quad \beta_{\gamma}=0.270949 .
\end{aligned}
$$

Значения (34) вместе образуют вектор параметров модели $\mathbf{p}_{\text {opt }}$, а подбор параметров баллистической модели на этом завершается.

Остаточные дисперсии $D_{1}$ и $D_{2}$ из (27) имеют следующие значения:

$$
D_{1}=184.148, \quad D_{2}=1.40754 \text {. }
$$

Это значит, что при уровне значимости более $99.999 \%$ принимается гипотеза о необходимости учета угла атаки в расчете.

На рис.1 сплошной линией обозначена зависимость эффективного баллистического коэффициента от угла бросания, рассчитанная по модели с подобранными выше параметрами. Для вычисления этой зависимости для каждой дальности из таблицы стрельбы проведено два модельных расчета с учетом угла атаки и без него. При расчете без учета угла атаки вычислялся эффективный баллистический коэффициент, дающий такой же угол бросания для данной дальности, что и с учетом угла атаки. На рис.1 кривая, полученная исходя из таблиц стрельбы (пунктирная линия), и модельная кривая (сплошная линия) оказались довольно близки в той области, где табличные данные достоверны, что говорит о том, что процесс подбора параметров модели прошел успешно.

Подбор параметров для пулемета Никитина, Соколова и Волкова (НСВ) калибром 12.7 мм с пулей Б-32 [13] дает следующий результат:

$$
\begin{aligned}
& \beta_{r}=0.173391, \quad \beta_{n m}=0.0452098, \quad \beta_{\alpha}=-2.24896 e-05, \\
& i=1.06284, \beta_{\delta}=0, \quad \beta_{\gamma}=0 .
\end{aligned}
$$

При этом остаточные дисперсии $D_{1}=7.433 e-3$ и $D_{2}=8.152 e-3$, так что гипотеза о необходимости учета угла атаки отвергается при уровне значимости $50 \%$. 
Заключение. Представлена полуэмпирическая баллистическая модель с четырьмя степенями свободы и подробно рассмотрен процесс оптимизации ее параметров. Данная модель позволяет рассчитать деривацию и учитывает влияние угла атаки снаряда на его лобовое сопротивление, при этом она требует лишь немного больше машинного времени, чем двумерная баллистическая модель $[1,5]$, в которой эффекты, связанные с вращением снаряда, не учитываются совсем. Поэтому она с успехом может применяться для вычисления баллистических поправок в реальном масштабе времени без использования заранее рассчитанных таблиц.

Для выполнения практических расчетов необходимо знание эталонной функции сопротивления $c_{x}$, аппроксимацию которой можно найти в [8-10, 14], учесть зависимость плотности среды $\rho$ от параметров атмосферы (температура, давление, влажность) и высоты полета, влияние ветра, а также зависимость начальной скорости от температуры заряда и массы снаряда. Данные вопросы подробно рассмотрены в [1, 5, 15-17].

Задача определения баллистических поправок является краевой задачей для системы дифференциальных уравнений внешней баллистики. Для ее решения используются различные математические методы. Например, можно использовать метод однопараметрической стрельбы [18], как это сделано в [8]. Составление подробного обзора методов решения краевой задачи для системы дифференциальных уравнений внешней баллистики планируется на следующих этапах исследования.

\section{СПИСОК ЛИТЕРАТУРЫ}

1. McCoy R.L. Modern Exterior Ballistics: The Launch and Flight Dynamics of Symmetric Projectiles // Atglen, PA: Schiffer Publishing Ltd., 2012, 328 p. (Technology \& Engineering). ISBN 978-0-7643-3825-0.

2. Мельников П.Н., Терпигорев М.А. Расчеты траектории полета артиллерийского снаряда // Известия ТулГУ. Технические науки, 2014, вып.12, ч.2, 6 с.

Melnikov P.N., Terpigorev M.A. Raschety traektorii poleta artilleriiskogo snariada // Izvestiia TulGU. Tekhnicheskie nauki, 2014, vyp.12, ch.2, 6 s.

3. ГОСТ В 24288-80. Снаряды неуправляемые артиллерийские, реактивные, активнореактивные. Метод расчета траектории полета. - М.: Изд-во стандартов, 1980, 55c.; GOST V 24288-80. Snariady neupravliaemye artilleriiskie, reaktivnye, aktivno-reaktivnye. Metod rascheta traektorii poleta. - M.: Izdatelstvo standartov, 1980, 55s.

4. https://www.hornady.com/

5. Коновалов А.А., Николаев Ю.В. Внешняя баллистика. - М.: ЦНИИ информации, 1979, 228c.

Konovalov A.A., Nikolaev IU.V. Vneshniaia ballistika. - M.: TSNII informatsii, 1979, 228s.

6. Окунев Б.Н. Вращательное движение артиллерийского снаряда. - М.- Л.: Государственное издательство технико-теоретической литературы, 1943, 120 с.

Okunev B.N. Vrashchatelnoe dvizhenie artilleriiskogo snariada. - M.- L.: Gosudarstvennoe izdatelstvo tekhniko-teoreticheskoi literatury, 1943, $120 \mathrm{~s}$. 
7. Шапиро Я.М. Внешняя баллистика. - М.: Оборонгиз, 1946, 408 с.; Shapiro IA.M. Vneshniaia ballistika. - M.: Oborongiz, 1946, 408 s.

8. Козлитин И.А., Омельянов А.С. Метод построения гладкой аппроксимации законов сопротивления // Математическое моделирование, 2016, т.28, № 10, с.23-32;

Kozlitin I.A., Omelianov A.S. Metod postroeniia gladkoi approksimatsii zakonov soprotivleniia // Matematicheskoe modelirovanie, 2016, t.28, №10, s.23-32.

9. Хайков В.Л. Обзор аналитических выражений закона сопротивления воздуха. 1943, Часть 1. // Электр. информ. сис. 2017, №4(15), с.74-90. ISSN 2312-9719.

Khaikov V.L. Obzor analiticheskikh vyrazhenii zakona soprotivleniia vozdukha. 1943, Chast 1 // Elektr. inform. sis., 2017, №4(15), s.74-90. ISSN 2312-9719.

10. Хайков В.Л. Обзор аналитических выражений закона сопротивления воздуха. 1943, Ч.2. // Электр. информац. системы, 2018, №1(16), с.85-97. ISSN 2312-9719.

Khaikov V.L. Obzor analiticheskikh vyrazhenii zakona soprotivleniia vozdukha. 1943 g. Chast 2. // Elektr. informatsionnye sistemy. 2018, №1(16), s.85-97. ISSN 2312-9719.

11. Кобзарь А.И. Прикладная математическая статистика. Для инженеров и научных работников. - М.: ФИЗМАТЛИТ, 2006, 816 с. ISBN 5-9221-0707-0.

Kobzar A.I. Prikladnaia matematicheskaia statistika. Dlia inzhenerov i nauchnykh rabotnikov. - M.: FIZMATLIT, 2006, 816 s. ISBN 5-9221-0707-0.

12. Руководство по 30-мм автоматическому гранатомёту на станке (АГС-17). Изд. 2-ое, стереотипное. - М.: Военное издательство МО СССР, 1982, 193 с.

Rukovodstvo po 30-mm avtomaticheskomu granatometu na stanke (AGS-17). Izdanie vtoroe, stereotipnoe. - M.: Voennoe izd-vo MO SSSR, 1982, $193 \mathrm{s.}$

13. Руководство по 12.7-мм пулемёту Утес (НСВ-12,7). - М.: Военное изд-во, 1986. Rukovodstvo po 12.7-mm pulemetu Utes (NSV-12,7). - M.: Voennoe izd-vo, 1986.

14. Ефремов А.К. Аппроксимация закона сопротивления воздуха 1943г. - М.: Наука и образование: Электрон. научно-техн. журн. МГТУ им. Н.Э. Баумана, 2013, вып.10, с.269-284. Efremov A.K. Approksimatsiia zakona soprotivleniia vozdukha 1943g. - M.: Nauka i obrazovanie:Elektronnyi nauchno-tekhn. zhurn. MGTU im. N.E. Baumana, 2013, vyp.10, s.269-284.

15. Бурлов В.В. и др. Баллистика ствольных систем. Под ред. Л.Н. Лысенко и А.М. Липанова. - М.: Машиностроение, 2006, 461 с.;

Burlov V.V. $i d r$. Ballistika stvolnykh sistem. Pod red. L.N. Lysenko i A.M. Lipanova. M.: Mashinostroenie, 2006, $461 \mathrm{~s}$.

16. Дмитриевский А.А., Лысенко Л.Н. Внешняя баллистика, 4-е изд., перераб. и доп. М.: Машиностроение, 2005, 608 с. ISBN 5-217-03252-9.

Dmitrievskii A.A., Lysenko L.N. Vneshniaia ballistika, 4-e izd., pererab. i dop. - M.: Mashinostroenie, 2005, 608 s. ISBN 5-217-03252-9.

17. ГОСТ 4401-81 Атмосфера стандартная. Параметры. ИПК Изд-во стандартов, 2004, 181с.; GOST 4401-81 Atmosfera standartnaia. Parametry. IPK Izd-vo standartov, 2004, 181s.

18. Калиткин Н.Н., Корякин П.В. Численные методы. В 2 кн. Кн.2: Методы математической физики. - М.: Академия, 2013, 304 с.;

Kalitkin N.N., Koriakin P.V. Chislennye metody. V 2 kn. Kn. 2: Metody matematicheskoi fiziki. - M.: Akademiia, 2013, 304 s. 